\title{
RADIAL PN JUNCTION NANOROD SOLAR CELLS: DEVICE PHYSICS PRINCIPLES AND ROUTES TO FABRICATION IN SILICON
}

\author{
Brendan M. Kayes, Christine E. Richardson, Nathan S. Lewis, Hany A. Atwater \\ California Institute of Technology, Pasadena, CA 91125
}

\begin{abstract}
We have developed quantitative device-physics models for a radial pn junction nanorod solar cell, that is, a cell which consists of densely packed nanorods attached to a conducting substrate, each nanorod with a pn junction in the radial direction. It is found that this novel design shows large improvements over the planar geometry so long as two conditions are satisfied: a) a planar solar cell made from the same material is collection limited, i.e. the diffusion length of minority carriers is too low to allow for collection of most or all of the light-generated carriers in the conventional planar geometry, and b) recombination in the depletion region is not too high, or, equivalently, the lifetime of carriers in the depletion region is not too short. In order to experimentally validate this concept, the vaporliquid-solid (VLS) growth of silicon (Si) nanorods has been explored using metal catalyst particles that are not as deleterious to the minority carrier lifetime of Si as gold (Au), the most commonly used wire growth catalyst.
\end{abstract}

\section{INTRODUCTION}

Inexpensive semiconductor absorber materials for use in photovoltaic applications generally have either a high level of impurities or a high density of defects, resulting in low minority carrier diffusion lengths [1]. Use of low diffusion length materials as the absorbing base in a conventional planar pn junction solar cell geometry results in devices having carrier collection limited by minority carrier diffusion in the base region. Increasing the thickness of the base in such a cell will therefore produce more light absorption but will not result in an increase in device efficiency. In the absence of sophisticated light trapping schemes, materials with low diffusion lengths and low absorption coefficients therefore cannot readily be incorporated into high energy conversion efficiency planar solar cell structures [2].

Radial pn junction nanorod cells provide a potential solution to this problem. A nanorod with a pn junction in the radial direction allows for separation of the requirements of light absorption and carrier extraction in two orthogonal directions. The cell can be thick in one dimension for optimal light absorption, while thin in another, for optimal carrier collection (see Fig. 1).

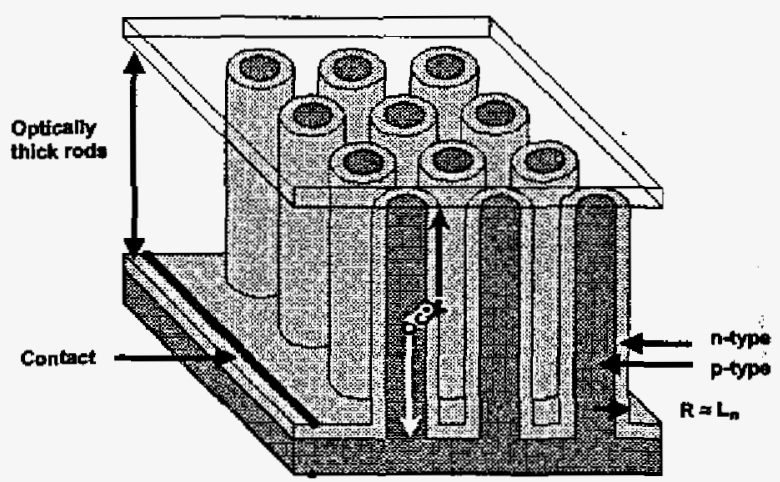

Fig. 1. Schematic and cross-section of the radial pn junction nanorod cell. Light is incident on the top surface. The light grey area is n-type, the dark grey area p-type. .

\section{DEVICE PHYSICS ANALYSIS}

The nanorod cell was analyzed by solving the semiconductor drift/diffusion carrier transport equations in cylindrical polar coordinates and comparing the result with the 1-d transport equation treatment for a convertional planar-junction cell. Simplifying assumptions necessary to obtain analytic solutions to the problem were made. The model was then applied to real materials, silicon (Si) and gallium arsenide (GaAs) (GaAs results are not included here - see [3] for details), using a $100 \mathrm{~mW} / \mathrm{cm}^{2}$, AM1.5 spectrum, to obtain quantitative comparisons of the two geometries. Cell efficiencies, open-circuit voltages, shortcircuit currents, and fill factors were calculated as a function of cell thickness (which is the same as the rod length in the nanorod case), nanorod radius, and electron diffusion length, at fixed emitter and base doping levels (or core and sheath dopings in the nanorod case) and window-layer thickness. Two regimes were considered first the trap density was assumed to be the same throughout the material and thus the quasi-neutral region and depletion region lifetimes were coupled, and secondly the depletion region trap density was held fixed at $10^{14} \mathrm{~cm}$ ${ }^{3}$, so that depletion region lifetimes $\tau_{n 0}, T_{p 0}=1 \mu s$, while the quasi-neutral region trap density was allowed to vary independently. Thus the relative impact of traps in the depletion- and quasi-neutral regions could be seen. 
The model for the radial pn junction nanorod solar cell was constructed by extending the analysis of the planar cell geometry [4] to a cylindrical geometry. The $p n$ junction In the nanorod was assumed to be abrupt, and the depletion approximation was assumed to be valid. The emitter layer (i.e. the exterior "shell" of the nanorod) was n-type, while the base (i.e. the interior "core" of the nanorod) was p-type. Light was normally incident on the top face of the nanorod. Recombination was assumed to purely be due to Shockley-Read-Hall recombination from a single trap level at mid-gap [5]; other recombination processes such as Auger recombination were neglected. Surface recombination effects were, however, included.

To simplify the analysis and to allow for analytic solutions, carrier transport was taken to be purely radial. Hence, each cross-sectional "slice" of the rod was treated separately, and the resulting flux was integrated over the length of the rod. This approximation is valid when the carrier motion in the z-direction is much less than the carrier motion in the r-direction, which is the case when the carrier concentration gradient and/or the electric field in the $r$-direction is much greater than in the z-direction. This condition is appropriate for materials that are collection-limited, because the length scale associated with carrier transport will be much shorter than that associated with light absorption.

No correction was made for less than $100 \%$ packing density. In practice light will not be incident truly normal to the rods, but rather at some angle. At small angles from normal incidence, light that is incident on the top of a rod will remain within the rod due to total internal reflection, while light that is not incident on the top of a rod is likely to pass through many rods while traversing the cell, due to the high aspect ratio of the rods. The precise details await further study.

Detailed results of this modeling are presented elsewhere [3], herein is a summary of the most important findings. It was found that this novel design shows large improvements over the planar geometry so long as two conditions are satisfled: a) a planar solar cell made from the same material is collection limited, i.e. the diffusion length of minority carriers is too low to allow for collection of most or all of the light-generated carriers in the conventional planar geometry, and b) recombination in the depletion region is not too high, or, equivalently, the lifetime of carriers in the depletion region is not too short. For $\mathrm{Si}$, this means that the lifetime of carriers in the depletion region lifetimes must be greater than $-10 \mathrm{~ns}$, or, equivalently, that the trap density in the depletion region must be less than $-10^{16} \mathrm{~cm}^{-3}$. If condition (a) is satisfied but not (b), then the radial cell outperforms the planar cell only slightly.

The short-circuit current density in the Si nanorod cell is essentially independent of the trap density in stark contrast to the planar cell, indicating that the radial pn junction design overcomes the problems of carrier collection that are present in the conventional planar geometry.
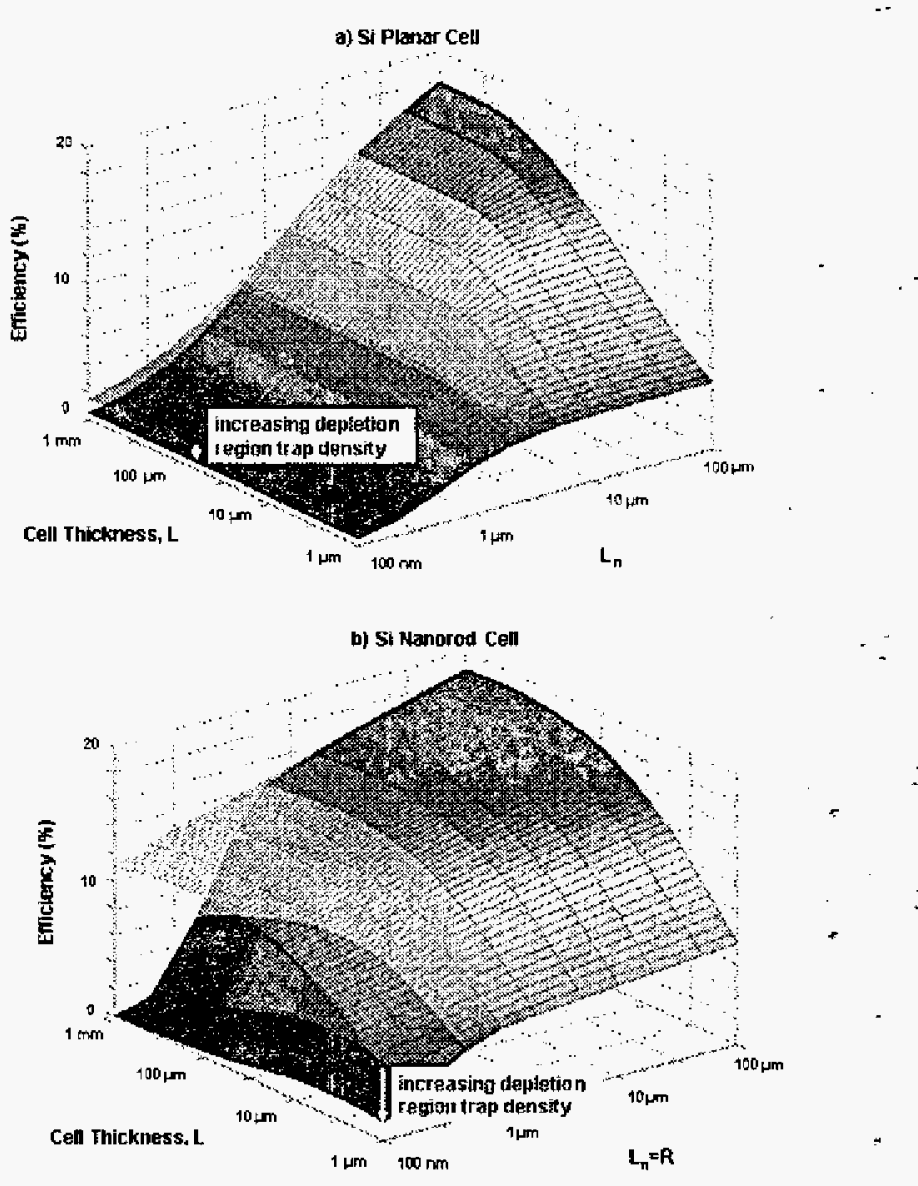

Fig. 2. Efficiency vs. cell thickness $L$ and minority electron diffusion length $L n$, for (a) a conventional planar pn junction Si cell, and (b) a radial pn junction nanorod. SI cell. In both cases the top surface shown in the plot has the depletion region trap density fixed at $10^{14} \mathrm{~cm}^{-3}$, so that depletion region lifetimes $T_{n 0}, T_{p 0}=1 \mu \mathrm{s}$, while the bottom surface has depletion region trap density equal to the trap density in the quasi-neutral region, at each value of $L_{n}$. In the radial pn junction nanorod case, the cell radius $R$ is set equal to $L_{n}$, a condition that was found to be near-optimal.

The degree to which the open-circuit voltage varied with the trap density depended most strongly on the trap density in the depletion region, for both the radial pn junction case and for the planar case. A low trap density in the depletion region produced a relatively high opencircuit voltage even if the quasi-neutral region trap density was relatively high. A high trap density $\left(>-10^{16} \mathrm{~cm}^{-3}\right)$ in the depletion region inevitably led to a low open-circuit voltage.

Taken together, this means that the efficiency of a radial pn junction nanorod solar cell can remain high despite a high quasi-neutral region trap density, provided that the depletion region trap density is not too high (Fig. 2). In Si with very low diffusion lengths $\left(L_{n}=100 \mathrm{~nm}\right)$, 
extremely large efficiency. gains (from $1.5 \%$ to $12 \%$ ) are possible by exploiting the radial pn junction nanorod geometry, provided that the trap density in the depletion region is held fixed at a relatively low level $<-5 \times 10^{15}$ $\mathrm{cm}^{-3}$ ). Even at higher depletion region trap densities (2.5x $10^{16} \mathrm{~cm}^{-3}$ ), in Si with $L_{n}=1 \mu \mathrm{m}$ efficiency gains from $4.2 \%$ to $13 \%$ are predicted. These increases are attributed to the greatly enhanced carrier collection that is possible in the radial pn junction nanorod cell, and is computational verification of the principle of exploiting geometry to extract carriers from optically thick, low diffusion-length solar cell materials.

An optimally designed radial pn junction nanorod cell should be doped as highly as possible in both $n$ - and ptype regions, have a narrow emitter width, and have a radius approximately equal to the diffusion length of electrons in the p-type "core" (in qualitative agreement with [6]). If light trapping techniques are not employed the nanorod length should be approximately equal to the "optical thickness" of the material, defined as the thickness of material required to absorb $90 \%$ of the power due to above-bandgap photons, which is $125 \mu \mathrm{m}$ for Si.

This large value for optical thickness is one reason that $\mathrm{Si}$ is a good candidate material for the nanorod cell. Si's large optical thickness implies that Si used in a conventional planar pn junction cell must have a large minority-carrier diffusion length in the base region.

\section{EXPERIMENTAL}

In order to realize a radial pn junction nanorod solar cell experimentally, routes to the fabrication of denselypacked, well-oriented arrays of nanorods, with diameters in the range $100 \mathrm{~nm}-1 \mu \mathrm{m}$, and lengths $-100 \mu \mathrm{m}$ (potentially less with light trapping), are of interest. Of particular interest is the Vapor-Liquid-Solid (V.S) method to fabricate Si nanorods on a $<111>$ Si substrate [7] [8]. A thermal Chemical Vapor Deposition (CVD) system was used, with silane $\left(\mathrm{SiH}_{4}\right)$ as the vapor-phase reactant, and, in some experiments, hydrogen $\left(\mathrm{H}_{2}\right)$ as a carrier gas. Metal seed particles were created either lithographically using a lift-off process, or by simple evaporation of a thin $(5 \mathrm{~nm})$ layer of metal that dewetted from the substrate during deposition to form an array of nanoparticles.

In choosing a catalyst particle to seed nanorod growth, one has to take many factors into account, particularly if one is concerned with making devices. Previously, gold (Au) has been used to seed the growth [9]. However, Au is known to form a deep-level trap state within the band-gap of Si [10]. Although Au has a relatively low solubility in the growing nanorod (segregation coefficient $2.5 \times 10^{-5}$ [4]), this nonetheless implies that Au concentrations of $-10^{18} \mathrm{~cm}^{-3}$ will be present in nanorods (segregation coefficient $2.5 \times 10^{-5} \times$ solubility of $\mathrm{Au}$ in $\mathrm{Si}$ at eutectic point 0.814 [11] $\times 5.0 \times$ $10^{22} \mathrm{Si}$ atoms $\mathrm{cm}^{-3}[10]$ ). This can create a high enough trap density to seriously degrade the electronic performance of Si, leading to sub-nanosecond lifetimes [10].
We have explored altemative catalysts that do not have such adverse effects on the minority carrier lifetime of Si. In searching for a catalyst one must take the following issues into account:

- Rod growth must be thermodynamically favorable [12]

- The catalyst should not form a deep-level trap state within the band-gap of $\mathrm{Si}$

- Si should be highly soluble in the liquid catalyst

- The catalyst should have low solubility in solid Si

- The catalyst-Si eutectic temperature should be matched to the vapor precursor decomposition kinetics

- The catalyst should not form an oxide, which would impede VLS nanorod growth

- The catalyst should have a low vapor pressure at the eutectic temperature, so that catalyst is not lost to the vapor phase

- The catalyst-Si binary phase diagram should not include solid intermediate phases, which could interfere with VLS growth of Si nanorods

- The catalyst should be inert to the chemical reaction products

Taking these considerations into account, aluminum (A), indium (In), and gallium (Ga) have been explored as catalysts for the VLS process. These elements have the advantage over Au of not forming deep-level traps within the band-gap of Si. The major disadvantage of these catalysts is their relatively higher solubility in solid $\mathrm{Si}$ (segregation coefficients for $\mathrm{Al}$, In and $\mathrm{Ga}$ are 0.002 , 0.0004 , and 0.008 , respectively [4]). However, since a ptype Si nanorod is ultimately desired this may not be a significant problem, since these impurities all form relatively shallow acceptor states near the valence band in Si [10].

Representative images of our fabrication results from In catalysts are shown in Fig. 3. The rod density as well as the range of rod diameters $(-100 \mathrm{~nm}$ to $>1 \mu \mathrm{m})$ are in the size range desired for nanorod solar cell applications positive signs for the use of In as a catalyst.

To date Al catalysts have yielded thin shells at the edges of the seed particles rather than nanorods. Also, the growth is much slower than typically seen in the VLS process. This is attributed to the aluminum oxide that forms very rapidly on the Al surface and acts as a barrier to VLS growth [13].

In order to fabricate the device pictured schematically in Fig. 1, control of nanorod density and orientation must be much better than has been achieved to date. If precise control of nanorod position via a template-less growth mechanism proves challenging, it may be possible to use porous alumina to template VLS growth of a nanorod array [14] [15]. To do this, a porous alumina layer of the desired thickness could first be grown on top of silicon, then seed particles deposited within the pores, and then Si nanorods grown in the pores by the VLS process. Having fabricated a well-oriented, dense aray of p-type nanorods, radia: pn 
junctions could be formed for example by phosphorus diffusion doping.
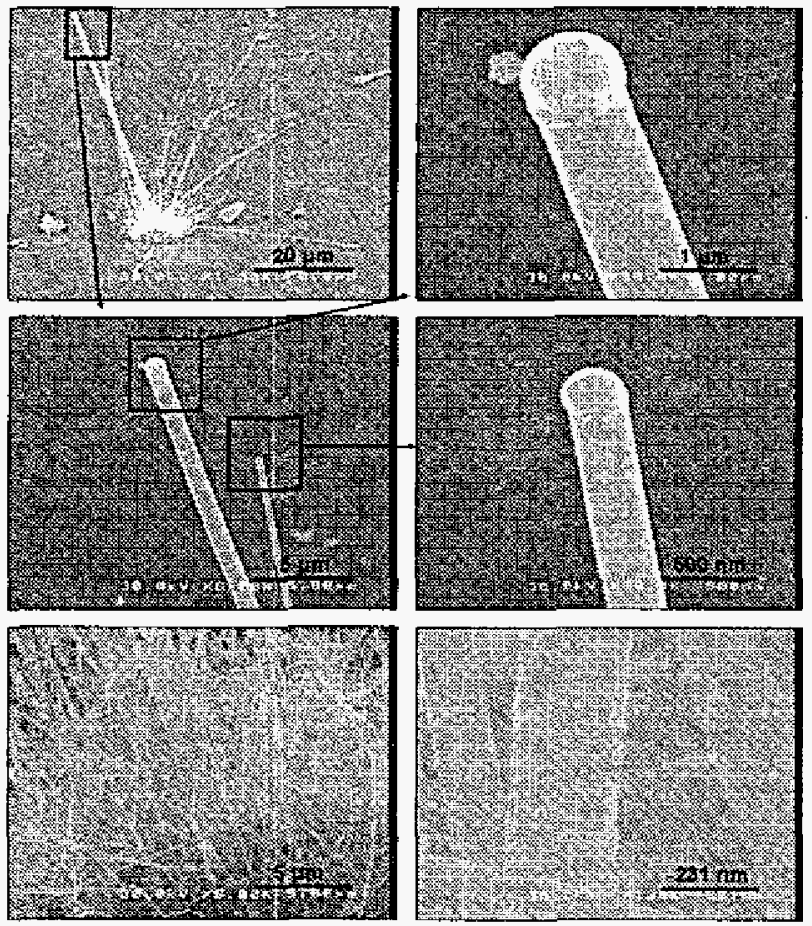

Fig. 3. Si nanorods grown from in seed particles, at pressure $P=1 \mathrm{Torr}, \mathrm{SiH}_{4}$ flow of $40 \mathrm{sccm}$, and temperature $\mathrm{T} \approx 500 \mathrm{C}$.

\section{CONCLUSIONS}

Radial pn junction nanorod cells are a novel approach to the creation of high efficiency solar cells using low lifetime materials. To attain high efficiency in this new geometry it is essential that the depletion region trap density is not too high $\left(<-10^{16} \mathrm{~cm}^{-3}\right)$. The trap density in the quasi-neutral regions can be higher than this with little detrimental effect.

Fabrication of a radial pn junction nanorod solar cell requires creating a dense array of well-oriented $\mathrm{Si}$ nanorods, each with a radial pn junction, as well contacts to both the $p$ - and n-type regions. Si nanorods have been grown by the VLS method using In catalyst particles, a step towards the fabrication of a nanorod solar cell.

\section{ACKNOWLEDGEMENTS}

The authors wouid like to thank BP Solar and NREL for their financial support.

\section{REFERENCES}

[1] V. Schlosser, "Limiting Factors for the Application of Crystalitine Upgraded Metallurgical Grade Silicon", IEEE Trans. Electron Devices 31, 1984. pp. 610-613.
[2] M. Imaizumi, T. Ito, M. Yamaguchi, and K. Kaneko, "Effect of Grain Size and Dislocation Density on the Performance of Thin Film Polycrystalline Silicon Solar Cells" J. Appl. Phys. 81, 1997, pp. 7635-7640.

[3] B.M. Kayes, N.S. Lewis, and H.A. Atwater, "Comparison of the device physics properties of planar and radial p-n junction nanorod solar celis", J. Appl. Phys. Submitted.

[4] A.L. Fahrenbruch, and R.H. Bube, Fundamentals of Solar Cells: Photovoltaic Solar Energy Conversion, Academic Press Ltd., Stanford, 1983, pp. 543-547 and 255.

[5] W. Shockley and W.T. Read, "Statistics of the Recombinations of Holes and Electrons" Phys. Rev. 87, 1952, pp. 835-842.

[6] B. Kannan, K. Castelino, and A. Majumdar, "Design of nanostructured heterojunction polymer photovoltaic devices", Nano Letters 3, 2003, pp. 1729-1733.

[7] R.S. Wagner and W.C. Ellis, "Vapor-Liquid-Solid Mechanism of Single Crystal Growth", Appl. Phys. Lett. 4, 1964, pp. 89-90.

[8] R.S. Wagner and W.C. Ellis, "The Vapor-Liquid-Solid Mechanism of Crystal Growth and Its Application to Silicon", Trans. Metall. Soc. AIME 233, 1965, pp. 10531064.

[9] M. Law, J. Goldberger, and P. Yang "Semiconductor Nanowires and Nanotubes", Annu. Rev. Mater. Res. 34, 2004, pp. 83-122.

[10] S.M. Sze, Physics of Semiconductor Devices, WileyInterscience, New York, 1969, pp. 21, 850, and 37.

[11] T. B. Massalski (ed.), Binary alloy phase diagrams, ASM International, Ohio, 1990 , p. 430.

[12] V.A. Nebol'sin and A.A. Shchetinin, "Role of Surface Energy in the Vapor-Liquid-Solid Growth of Silicon", Inorganic Materials 39, 2003, pp. 899-903.

[13] Y. Civale, L.K. Nanver, P. Hadley, E.J.G. Goudena, "Aspects of Silicon Nanowire Synthesis by AluminumCatalyzed Vapor-Liquid-Solid Mechanism", http://qt.tn.tudelft.nl/ -hadley/publications/safe/civalesafe2004.pdf

[14] J.S. Choi et al., "Monodisperse metal nanowire arrays on Si by integration of template synthesis with silicon technology", J. Mater. Chem. 13, 2003, pp. 11001103.

[15] K.K. Lew and J.M. Redwing, "Growth characteristics of silicon nanowires synthesized by vapor-liquid-solid growth in nanoporous alumina templates", Joumal of Crystal Growth 254, 2003, pp. 14-22. 